\title{
Some results on one-sided generalized Lie ideals with derivation
}

Neşet Aydin, Kâzim Kaya, and Öznur Gölbaşi 


\title{
SOME RESULTS ON ONE-SIDED GENERALIZED LIE IDEALS WITH DERIVATION
}

\author{
Neşet Aydin \\ Mersin University, Faculty of Arts and Science, Department of Mathematics \\ Mersin, Turkey \\ neseta@mersin.edu.tr \\ KÂZIM KAYA \\ 18 Mart University, Faculty of Arts and Science, Department of Mathematics \\ Çanakkale, Turkey \\ kkaya@comu.edu.tr \\ ÖZNUR GÖLBAŞI \\ Cumhuriyet University, Faculty of Arts and Science, Department of Mathematics \\ Sivas, Turkey \\ ogolbasi@cumhuriyet.edu.tr
}

[Received: June 6, 2001]

\begin{abstract}
Let $R$ be a prime ring with a characteristic not equal to two, $\sigma, \tau$ be automorphisms of $R$, and $d$ be a nonzero derivation of $R$ commuting with $\sigma$ and $\tau$. It is proved that for any $(\sigma, \tau)$-left Lie ideal $U$ of $R$ : (1) if $d(U) \subseteq Z$, then $\sigma(u)+\tau(u) \in Z$, for all $u \in U$, (2) if $d^{2}(U)=0$, then $\sigma(u)+\tau(u) \in Z$, for all $u \in U$, (3) if char $R \neq 2,3, d(U) \subseteq U$ and $d^{2}(U) \subseteq Z$, then $\sigma(u)+\tau(u) \in Z$, for all $u \in U$.
\end{abstract}

Mathematical Subject Classification: 16N60, 16W25, 16A72, 16U80

Keywords: prime ring, Lie ideal, generalized Lie ideal, derivation

\section{Introduction}

Let $R$ be a ring and $\sigma, \tau$ be two mappings from $R$ into itself. We write $[x, y]$, $[x, y]_{\sigma, \tau}$ for $x y-y x$ and $x \sigma(y)-\tau(y) x$, respectively, and make extensive use of basic commutator identities: $(x y, z)=x[y, z]+(x, z) y=x(y, z)-[x, z] y,[x y, z]_{\sigma, \tau}=$ $x[y, z]_{\sigma, \tau}+[x, \tau(z)] y=x[y, \sigma(z)]+[x, z]_{\sigma, \tau} y$.

An additive mapping $D: R \rightarrow R$ is called a derivation if $D(x y)=D(x) y+x D(y)$ holds for all $x, y \in R$. A derivation $D$ is inner if there exists an $a \in R$ such that $D(x)=[a, x]$ holds for all $x \in R$..

For subsets $A, B \subset R$, let $[A, B]\left([A, B]_{\sigma, \tau}\right)$ be the additive subgroup generated by all $[a, b]\left([a, b]_{\sigma, \tau}\right)$ for all $a \in A$ and $b \in B$. We recall that in a Lie ideal, $L$ is 
an additive subgroup of $R$ such that $[R, L] \subset L$. We first introduce the generalized Lie ideal in [6] as follows. Let $U$ be an additive subgroup of $R, \sigma, \tau: R \rightarrow R$ two mappings. Then $(i) U$ is a $(\sigma, \tau)$-right Lie ideal of $R$ if $[U, R]_{\sigma, \tau} \subset U$. (ii) $U$ is a $(\sigma, \tau)$-left Lie ideal of $R$ if $[R, U]_{\sigma, \tau} \subset U$. (iii) $U$ is both a $(\sigma, \tau)$-right Lie ideal and $(\sigma, \tau)$-left Lie ideal of $R$ then $U$ is a $(\sigma, \tau)$-Lie ideal of $R$. Every Lie ideal of $R$ is a $(1,1)$-left Lie ideal of $R$, where $1: R \rightarrow R$ is the identity map. As an example, let $I$ be the set of integers,

$$
\begin{aligned}
& R=\left\{\left(\begin{array}{ll}
x & y \\
z & t
\end{array}\right) \mid x, y, z, t \in I\right\}, \\
& U=\left\{\left(\begin{array}{ll}
x & y \\
0 & x
\end{array}\right) \mid x, y \in I\right\} \subset R,
\end{aligned}
$$

and $\sigma, \tau: R \rightarrow R$ the mappings defined by $\tau(x)=a x a, \sigma(x)=b x b^{-1}$, where $a=$ $\left(\begin{array}{ll}1 & -1 \\ 0 & -1\end{array}\right)$ and $b=\left(\begin{array}{ll}1 & 2 \\ 0 & 1\end{array}\right) \in R$. Then $U$ is a $(\sigma, \tau)-$ left Lie ideal but not a Lie ideal of R. Some algebraic properties of $(\sigma, \tau)$-Lie ideals are considered in [2], [3] and [6], where further references can be found.

Let $R$ be a prime ring with a characteristic not equal to two, $d: R \rightarrow R$ a nonzero derivation of $\mathrm{R}$ and $U$ a Lie ideal of $R$. In [5] Bergen at all state that if $d^{2}(U)=0$, then $U \subset Z$. Lee and Lee extended this result that if $d^{2}(U) \subset Z$, then $U \subset Z$ in [4]. Let $d$ be a nonzero derivation such that $\sigma d=d \sigma, \tau d=d \tau$ and $U$ a $(\sigma, \tau)$-Lie ideal of $R$. Aydın and Soytürk [3] proved that if $d^{2}(U)=0$, then $U \subset Z$. In the present paper, we generalize this result on $(\sigma, \tau)$-left Lie ideal of $R$. Furthermore, we shall extend this theorem by proving that $d^{2}(U) \subset Z$ then $\sigma(u)+\tau(u) \in Z$, for all $u \in U$ in the case of a characteristic not equal to two and three.

Throughout, $R$ will represent a prime ring with a characteristic not equal to 2 with automorphisms $\sigma, \tau$ and non-zero derivation $d$ such that $\sigma d=d \sigma, \tau d=d \tau$ and $Z$ the center of $R, U$ a $(\sigma, \tau)$-left Lie ideal of $R$. Further, we often use the relations:

$$
[x y, z]_{\sigma, \tau}=x[y, z]_{\sigma, \tau}+[x, \tau(z)] y=x[y, \sigma(z)]+[x, z]_{\sigma, \tau} y .
$$

\section{Results}

Lemma 1. Let $U$ a $(\sigma, \tau)$-left Lie ideal of $R . d^{2}(U)=0$ and $d(U) \subset Z$ then $\sigma(u)+$ $\tau(u) \in Z$, for all $u \in U$.

Proof. If $U \subset Z$, then the proof is obvious. So, we assume that $U \not \subset Z$. For any $u \in U$ and $x \in R, \tau(u)[x, u]_{\sigma, \tau}=[\tau(u) x, u]_{\sigma, \tau}+[\tau(u), \tau(u)] x \in U$. By hypothesis, $0=d^{2}\left(\tau(u)[x, u]_{\sigma, \tau}\right)=d\left(d(\tau(u))[x, u]_{\sigma, \tau}+\tau(u) d\left([x, u]_{\sigma, \tau}\right)\right)=2 d(\tau(u)) d\left([x, u]_{\sigma, \tau}\right)$. Since $\operatorname{char} R \neq 2$, we obtain $d(\tau(u)) d\left([x, u]_{\sigma, \tau}\right)=0$, for all $x \in R, u \in U$. Because of $d(U) \subset Z$ we have,

$$
d(u)=0 \quad \text { or } \quad d\left([x, u]_{\sigma, \tau}\right)=0 \quad \forall x \in R, u \in U .
$$

Assume $d(u) \neq 0$. Then $d\left([x, u]_{\sigma, \tau}\right)=0$, for all $x \in R$. Writing $x \sigma(u)$ by $x$ in this equation, $0=d\left([x \sigma(u), u]_{\sigma, \tau}\right)=d\left([x, u]_{\sigma, \tau} \sigma(u)\right)=d\left([x, u]_{\sigma, \tau}\right) \sigma(u)+[x, u]_{\sigma, \tau} d(\sigma(u))$ 
we obtain

$$
[x, u]_{\sigma, \tau} d(\sigma(u))=0 \quad \forall x \in R .
$$

Substituting $x y, y \in R$ for $x$ in $(2.2)$, we have $0=[x y, u]_{\sigma, \tau} d(\sigma(u))=x[y, u]_{\sigma, \tau} d(\sigma(u))+$ $[x, \tau(u)] y d(\sigma(u))$ and so,

$$
[R, \tau(u)] R d(\sigma(u))=0
$$

By primeness of $R$, we obtain $u \in Z$. Thus, if we return to (2.1), then we get

$$
d(u)=0 \quad \text { or } \quad u \in Z .
$$

Now, let us define the subsets $L=\{u \in U \mid u \in Z\}$ and $K=\{u \in U \mid d(u)=$ $0\}$. Clearly, each $L$ and $K$ is an additive subgroup of $U$. Moreover, $U$ is the settheoretic union of $L$ and $K$. But a group cannot be the set-theoretic union of two proper subgroups, hence $L=U$ or $K=U$. In the former case, $U \subset Z$, which is a contradiction. Therefore, it must be $d(U)=0$ and so,

$$
0=d\left([x, u]_{\sigma, \tau}\right)=[d(x), u]_{\sigma, \tau} \quad \text { for all } x \in R, u \in U .
$$

By [7, Lemma 1], we obtain $\sigma(u)+\tau(u) \in Z$, for all $u \in U$. Hence the proof is complete.

Theorem 1. Let $U a(\sigma, \tau)$-left Lie ideal of $R$. If $d(U) \subset Z$ then $\sigma(u)+\tau(u) \in Z$, for all $u \in U$.

Proof. Assume that $U \not \subset Z$. For any $x, y \in R$ and $u, v \in U$, by hypothesis, $d\left([d(v) x, u]_{\sigma, \tau}\right)$ $=d\left(d(v)[x, u]_{\sigma, \tau}+[d(v), \tau(u)] x\right)=d\left(d(v)[x, u]_{\sigma, \tau}\right) \in Z$ and so,

$$
d^{2}(v)[x, u]_{\sigma, \tau}+d(v) d\left([x, u]_{\sigma, \tau}\right) \in Z
$$

Since $Z$ is a subring of $R$ and $d(U) \subset Z$, we have

$$
d^{2}(v)[x, u]_{\sigma, \tau} \in Z \quad \forall x \in R, u, v \in U .
$$

Replacing $x$ by $x \sigma(u), u \in U$ in (2.3) and applying the above argument, we obtain

$$
d^{2}(v)[x, u]_{\sigma, \tau} \sigma(u) \in Z \quad \forall x \in R, u, v \in U .
$$

Since $d^{2}(v)[x, u]_{\sigma, \tau} \in Z$ and $R$ is prime ring, we get

$$
d^{2}(v)[x, u]_{\sigma, \tau}=0 \quad \text { or } \quad u \in Z .
$$

If $d^{2}(v)[x, u]_{\sigma, \tau}=0$ for all $x \in R$. In this equation by taking $x y, y \in R$ for $x$ and using this equation, we have $0=d^{2}(v)[x y, u]_{\sigma, \tau}=d^{2}(v)[x, u]_{\sigma, \tau} y+d^{2}(v) x[y, \sigma(u)]=$ $d^{2}(v) x[y, \sigma(u)]$. By the primeness of $R$, it implies that $d^{2}(U)=0$ or $U \subset Z$. In the former case, we get $\sigma(u)+\tau(u) \in Z$, for all $u \in U$ by Lemma 1 . Thus, we conclude that $\sigma(u)+\tau(u) \in Z$, for all $u \in U$.

Now, suppose that $U$ is a $(\sigma, \tau)$-left Lie ideal of $R$. Since for all $u, v \in U$ and $x \in R$,

$$
\begin{aligned}
{[x, d(u)+v]_{\sigma, \tau} } & =[x, d(u)]_{\sigma, \tau}+[x, v]_{\sigma, \tau} \\
& =[x, d(u)]_{\sigma, \tau}+[d(x), u]_{\sigma, \tau}-[d(x), u]_{\sigma, \tau}+[x, v]_{\sigma, \tau} \\
& =d\left([x, u]_{\sigma, \tau}\right)-[d(x), u]_{\sigma, \tau}+[x, v]_{\sigma, \tau} \in d(U)+U .
\end{aligned}
$$


We conclude that $d(U)+U$ is a $(\sigma, \tau)$-left Lie ideal of $R$. Furthermore, if $d^{2}(U)=0$ then $d(d(U)+U) \subset d(U) \subset d(U)+U$ and $d^{2}(d(U)+U)=0$. Therefore without losing generality, we may assume that if $U$ is a $(\sigma, \tau)$-left Lie ideal of such that $d^{2}(U)=0$, then $d(U) \subset U$.

Lemma 2. Let $U$ a $(\sigma, \tau)$-left Lie ideal of $R$. $d^{2}(U)=0$ and a be an element of $R$. If $a d\left([R, U]_{\sigma, \tau}\right)=0$, then $a=0$ or $\sigma(u)+\tau(u) \in Z$, for all $u \in U$.

Proof. For $x[\sigma(u), \sigma(u)]+[x, u]_{\sigma, \tau} \sigma(u)=[x \sigma(u), u]_{\sigma, \tau} \in[R, U]_{\sigma, \tau}$ by hypothesis $0=$ $a d\left([x, u]_{\sigma, \tau} \sigma(u)\right)=a d\left([x, u]_{\sigma, \tau}\right) \sigma(u)+a[x, u]_{\sigma, \tau} d(\sigma(u))$ and so

$$
a[x, u]_{\sigma, \tau} d(\sigma(u))=0, \forall x \in R, u \in U .
$$

Since $d^{2}(U)=0$, from the above remark we may assume $d(U) \subset U$. So, replacing $u+d(v), v \in U$ by $u$ in $(2.4)$

$$
0=a[x, u+d(v)]_{\sigma, \tau} d(\sigma(u+d(v)) .
$$

Expanding the last equation and using $d^{2}(U)=0, \sigma d=d \sigma$ and (2.4), we get $a[x, d(v)]_{\sigma, \tau} d(\sigma(u))=0$, for all $u, v \in U, x \in R$. That is,

$$
\sigma^{-1}\left(a[x, d(v)]_{\sigma, \tau}\right) d(U)=0 .
$$

By [1, Theorem 2] we have $\sigma(u)+\tau(u) \in Z$, for all $u \in U$ or $a[x, d(v)]_{\sigma, \tau}=0$. Replacing $x y, y \in R$ in the last equation, we obtain $a x[y, \sigma(d(v)]=0$. Since $R$ is a prime ring, we conclude $a=0$ or $d(U) \subset Z$. It gives $\sigma(u)+\tau(u) \in Z$, for all $u \in U$ from Theorem 1. This completes the proof.

Theorem 2. Let $U$ a $(\sigma, \tau)$-left Lie ideal of $R$. If $d^{2}(U)=0$ then $\sigma(u)+\tau(u) \in Z$, for all $u \in U$.

Proof. Assume that $U \nsubseteq Z$. There exists a $u_{0} \in U$ such that

$$
\sigma\left(u_{0}\right)+\tau\left(u_{0}\right) \notin Z .
$$

For $[x, u]_{\sigma, \tau} \sigma(u) \in U$,

$$
\begin{aligned}
0 & =d^{2}\left([x, u]_{\sigma, \tau} \sigma(u)\right) \\
& =d^{2}\left([x, u]_{\sigma, \tau}\right) \sigma(u)+2 d\left([x, u]_{\sigma, \tau}\right) d(\sigma(u))+[x, u]_{\sigma, \tau} d^{2}(\sigma(u)) .
\end{aligned}
$$

In view of the hypothesis and $\operatorname{char} R \neq 2$, we have

$$
d\left([x, u]_{\sigma, \tau}\right) d(\sigma(u))=0, \forall x \in R, u \in U .
$$

Similarly for $\tau(u)[x, u]_{\sigma, \tau} \in U$, we get

$$
d(\tau(u)) d\left([x, u]_{\sigma, \tau}\right)=0, \forall x \in R, u \in U .
$$

By hypothesis $0=d^{2}\left([u, v]_{\sigma, \tau}\right)=\left[d^{2}(u), v\right]_{\sigma, \tau}+2[d(u), d(v)]_{\sigma, \tau}+\left[u, d^{2}(v)\right]_{\sigma, \tau}$. Using $d^{2}(U)=0$ and $\operatorname{char} R \neq 2$, we obtain

$$
[d(u), d(v)]_{\sigma, \tau}=0, \forall u, v \in U .
$$

That is

$$
d(u) \sigma(d(v))=\tau(d(v)) d(u), \forall u, v \in U .
$$


Now, let us linearize (2.7) on $u=u+v$ and use (2.8), then we have

$$
d(\tau(u)) d\left([x, v]_{\sigma, \tau}+d(\tau(v)) d\left([x, u]_{\sigma, \tau}\right)=0, \forall x \in R, u, v \in U .\right.
$$

Multiply on the right by $d(\sigma(u))$ and use (2.8), (2.6), we obtain

$$
(d(\tau(u)))^{2} d\left([x, v]_{\sigma, \tau}\right)=0, \forall x \in R, u, v \in U .
$$

The last equation reduces to $(d(\tau(U)))^{2} d\left([R, U]_{\sigma, \tau}\right)=0$. By Lemma 2 and (2.5), we get $(d(U))^{2}=0$. Otherwise, writing $d(v)$ for $v$ in (2.9) and using $d \tau=\tau d$, we see that

$$
d(U) \tau^{-1}\left([d(x), d(v)]_{\sigma, \tau}\right)=0, \forall x \in R, v \in U .
$$

This means from $\left[1\right.$, Theorem 2] $\sigma(u)+\tau(u) \in Z$, for all $u \in U$ or $[d(x), d(v)]_{\sigma, \tau}=0$. By our assumption, we get $[d(x), d(v)]_{\sigma, \tau}=0$, for all $x \in R, v \in U$. If we write $x d(u), u \in$ $U$ for $x$ in the last equation, we have $0=\left[d(x d(u), d(v)]_{\sigma, \tau}=[d(x) d(u), d(v)]_{\sigma, \tau}=\right.$ $[d(x), \tau(d(v)] d(u)$ and so,

$$
[d(R), \tau(d(U))] d(U)=0 .
$$

From the above argument, we have $d(U) \subset Z$ by [1, Theorem 2]. That is $\sigma(u)+\tau(u) \in$ $Z$, for all $u \in U$ from Theorem 1 .

Theorem 3. Let $U a(\sigma, \tau)$-left Lie ideal of $R$ and char $R \neq 2,3$. If $d(U) \subset U$ and $d^{2}(U) \subset Z$, then $\sigma(u)+\tau(u) \in Z$, for all $u \in U$.

Proof. If $U \subset Z$, then the proof of the theorem is obvious. So, we assume that $U \nsubseteq Z$. That is,

$$
\sigma\left(u_{0}\right)+\tau\left(u_{0}\right) \notin Z, \exists u_{0} \in U .
$$

Suppose that $d(Z)=0$. Thus, we have

$$
d^{3}(U)=d\left(d^{2}(U)\right) \subset d(Z)=0 .
$$

Now, for $\tau(u)[x, u]_{\sigma, \tau} \in U$, where $x \in R$ and $u \in U$,

$$
\begin{aligned}
0 & =d^{3}\left(\tau(u)[x, u]_{\sigma, \tau}\right) \\
& =3\left(d^{2}(\tau(u)) d\left([x, u]_{\sigma, \tau}\right)+d(\tau(u)) d^{2}\left([x, u]_{\sigma, \tau}\right) .\right.
\end{aligned}
$$

Since $\operatorname{char} R \neq 3$, we get

$$
d^{2}(\tau(u)) d\left([x, u]_{\sigma, \tau}\right)+d(\tau(u)) d^{2}\left([x, u]_{\sigma, \tau}\right)=0 .
$$

Taking $d(u)$ by $u$ and using $\tau d=d \tau, d^{3}(U)=0$, we obtain

$$
d^{2}(\tau(u)) d^{2}\left([x, d(u)]_{\sigma, \tau}\right)=0 .
$$

Since $d^{2}(U) \subset Z$, the last equation gives us

$$
d^{2}(u)=0 \quad \text { or } \quad d^{2}\left([x, d(u)]_{\sigma, \tau}\right)=0 .
$$

Let us define $K=\left\{u \in U \mid d^{2}(u)=0\right\}$ and $L=\left\{u \in U \mid d^{2}\left([x, d(u)]_{\sigma, \tau}\right)=0, \forall x \in R\right\}$. Clearly, both $K$ and $L$ are additive subgroups of $U$. Moreover, $U$ is the set-theoretic union of $K$ and $L$. But a group cannot be the set-theoretic union of two proper subgroups, hence $K=U$ or $L=U$. If $K=U$ then $\sigma(u)+\tau(u) \in Z$, for all $u \in U$ by Theorem 2 and it contradicts (2.10). So, we get $L=U$. That is,

$$
d^{2}\left([x, d(u)]_{\sigma, \tau}\right)=0, \forall x \in R, u \in U .
$$


In this equation replace $x$ by $\tau(d(u)) x, u \in U, x \in R$, then we get

$$
\begin{aligned}
0 & =d^{2}\left(\tau(d(u))[x, d(u)]_{\sigma, \tau}\right) \\
& =\tau\left(d^{3}(u)\right)[x, d(u)]_{\sigma, \tau}+2 \tau\left(d^{2}(u)\right) d\left([x, d(u)]_{\sigma, \tau}\right)+\tau(d(u)) d^{2}\left([x, d(u)]_{\sigma, \tau}\right) .
\end{aligned}
$$

Using (2.11) and $d^{3}(U)=0, \operatorname{char} R \neq 2$, we obtain $\tau\left(d^{2}(u)\right) d\left([x, d(u)]_{\sigma, \tau}\right)=0$. Since $d^{2}(U) \subset Z$, we have

$$
d^{2}(u)=0 \quad \text { or } \quad d\left([x, d(u)]_{\sigma, \tau}\right)=0 .
$$

Let $K=\left\{u \in U \mid d^{2}(u)=0\right\}$ and $L=\left\{u \in U \mid d\left([x, d(u)]_{\sigma, \tau}\right)=0, \forall x \in R\right\}$. Each of $K$ and $L$ is an additive subgroup of $U$ such that $U=K \cup L$. The above trick gives us $U=K$ or $U=L$. In the former case, $d^{2}(U)=0$, which forces $\sigma(u)+\tau(u) \in Z$, for all $u \in U$ by Theorem 2, which is a contradiction. Thus $U=L$ and hence $d\left([x, d(u)]_{\sigma, \tau}\right)=0$ for all $u \in U$. Replacing $\tau(d(u)) x, u \in U, x \in R$ by $x$ we have $\tau\left(d^{2}(u)\right)[x, d(u)]_{\sigma, \tau}=0$. Since $d^{2}(U) \subset Z$, we obtain

$$
d^{2}(u)=0 \quad \text { or } \quad[x, d(u)]_{\sigma, \tau}=0 \text { for all } x \in R .
$$

Again applying the above trick, we obtain $[x, d(u)]_{\sigma, \tau}=0$. Taking $x y, y \in R$ in place of $x$ and using (2.12), we have

$$
0=[x y, d(u)]_{\sigma, \tau}=x[y, d(u)]_{\sigma, \tau}+[x, \sigma(d(u))] y=[x, \sigma(d(u))] y .
$$

Since $R$ is a prime ring, we obtain $d(U) \subset Z$. By Theorem 1, it gives $\sigma(u)+\tau(u) \in Z$, for all $u \in U$, which is a contradiction. Thus, in the case of $d(Z)=0$ the proof is completed.

Now, we would like to settle the problem when $d(Z)$ is different from zero. There is a non-zero $d(\alpha) \in d(Z)$ such that $\alpha \in Z$. In view of the hypothesis for $[\alpha x, u]_{\sigma, \tau}=$ $\alpha[x, u]_{\sigma, \tau} \in U$,

$$
d^{2}\left(\alpha[x, u]_{\sigma, \tau}\right)=d^{2}(\alpha)[x, u]_{\sigma, \tau}+2 d(\alpha) d\left([x, u]_{\sigma, \tau}\right)+\alpha d^{2}\left([x, u]_{\sigma, \tau}\right) \in Z .
$$

Since $d^{2}(U) \subset Z$, the third term is in the center of $R$. So, we get

$$
d^{2}(\alpha)[x, u]_{\sigma, \tau}+2 d(\alpha) d\left([x, u]_{\sigma, \tau}\right) \in Z, \forall x \in R, u \in U .
$$

Replace $x$ by $x \alpha$ in (2.13) to get

$$
\left(d^{2}(\alpha)[x, u]_{\sigma, \tau}+2 d(\alpha) d\left([x, u]_{\sigma, \tau}\right)\right) \alpha+2 d(\alpha)[x, u]_{\sigma, \tau} d(\alpha) \in Z .
$$

However, in view of (2.13) and $\alpha \in Z$, this equation reduces to $2 d(\alpha)[x, u]_{\sigma, \tau} d(\alpha) \in Z$. Since $R$ is a prime ring, $\operatorname{char} R \neq 2$ and $0 \neq d(\alpha) \in Z$, we have $[x, u]_{\sigma, \tau} \in Z$ for all $x \in R, u \in U$. By [8, Lemma 1], we obtain $\sigma(u)+\tau(u) \in Z$, for all $u \in U$. This completes the proof.

\section{REFERENCES}

[1] Aydin, N.: Notes on generalized Lie ideals, Analele Universitatii din Timisoara Seria, Matematica-Informatica-Vol., XXVI(2), (1999), 7-13.

[2] Aydin, N. and Kandamar, H.: $(\sigma, \tau)$-Lie ideals in prime rings, Doğa Tr. J. of Math., 18(2), (1994), 143-148. 
[3] Aydin, N. and SoytüRk, M.: $(\sigma, \tau)$-Lie ideals in prime rings with derivations, Doğa Tr. J. of Math. 19, (1993), 239-244.

[4] LeE, P. H. and Lee, T. K.: Lie ideals of prime rings with derivations, Bull. Inst. Math. Acad. Sinica., 11, (1983), 75-80.

[5] Bergen, J., Herstein, I. N. and Kerr, J. W.: Lie ideals and derivation of prime rings, J. of Algebra, 71, (1981), 259-267.

[6] KaYA, K.: $(\sigma, \tau)$-Lie ideals in prime rings, An. Univ. Timisoara Stiinte Math., 30(2-3), $251-255$.

[7] Kaya, K., Gölbaşi, Ö. and Aydin, N.: Some results for generalized Lie ideals in prime rings with derivation II., Applied Mathematics E-Notes, 1 (2001), 24-30.

[8] SoYtürk, M.: $(\sigma, \tau)$-Lie ideals in prime rings with derivations, Doğa Tr. J. of Math., 18, (1994), 280-283. 